\title{
Stanistaw Bylina
}

\section{La Bible, les laïcs et l'Eglise en Pologne entre le Moyen Age et la Réforme}

C'est non sans quelque crainte de voir mon sujet se situer en marge du présent colloque. Certes, la contestaton religieuse offrait souvent une référence aux relations entre les différents éléments de la triade du titre, mais dans le cas étudié, nous considérons ces relations dans le contexte d'un pays qui, jusqu'à la troisième décade du XVe siècle ne connut pas de mouvements hérétiques. C'est-à-dire jusqu'au temps où se firent entendre en Pologne les échos d'intensité modérée, encore que fort prolongés, de l'hussitisme tchèque.

Des tendances à une étude libre, non contrólée par l'Église, des textes bibliques, pouvaient se faire jour également hors des communautés hérétiques. Il est toutefois certain qu'elles s'affirmaient avec le plus de relief à travers les opinions de membres de ces communautés, ceux en particulier qui professaient la pauvreté volontaire ${ }^{1}$. Dans cet ordre d'idées, une interprétation par soi-même, le plus souvent au pied de la lettre, de l'Écriture Sainte, visait nécessairement à mettre en opposition une réalité évangélique érigée en idéal et de ce fait prêchée, avec celle de la vie ecclésiastique et sociale du temps, jugée passible de la plus âpre des critiques.

Or, en territoire du Royaume de Pologne réunifié, nous ne trouvons, au XIVe siècle, d'articulation des idées ci-des-sus par un valdéisme, inconnu sur les bords de la Vistule, pas plus que sur le plan d'une vie religieuse d'une orthodoxie sans histoires. Nous ignorons si, et dans quelle mesure, les laïss aspiraient à une connaissance plus poussée de l'Écriture Sainte que celle que leur offrait l'Église à travers les courtes lectures bibliques pendant les messes du dimanche et des jours de fête. Les fragments traduits du Nouveau Testament étaient destinés précisément à des fins liturgiques, ce qui les soustrayait à la lecture individuelle par les laïcs. Pour ce qui était de l'Ancien Testament, les seules traductions disponibles c'étaient des psautiers, utilisés comme livres de prière à la Cour et dans les couvents féminins ${ }^{2}$. Au XIVe siècle, en ont vraisemblablement bénéficié les pieuses

1 Tadeusz Manteuffel, Die Geburt der Ketzerei (Wien, Frankfurt, Zürich 1965) passim; Giovanni Gonnet, Amedeo Molnár, Les Vaudois au Moyen Age (Torino 1974) 197-202.

2 Aleksandra Witkowska, Biblia. Przekłady na język polski, dans: Encyklopedia Katolicka, t.2 (Lublin 1985) 409; Teresa Michatowska, Sredniowiecze (Wielka Historia Literatury Polskiej) (Warszawa 1995) 294-300. 
princesses de sang polonaises, et l'exécution du plus prestigieux d'entre eux (connu sous l'appellation de Psautier de Saint-Florien), serait le fait du mécénat de la reine de Pologne Hedvige d'Anjou.

En dépit de la rareté de documents relatifs aux phénomènes religieux de masse du XIVe siècle, l'on peut parler, sans risque d'un démenti, d'une non-préparation de la société polonaise d'alors à apprendre à connaître de la Bible ou, plus précisément, à en ressentir le besoin. L'Église, en instruisant et en éduquant les fidèles, était loin de stimuler un tel besoin. La prédication en Pologne, avant d'avoir été renforcée par les élèves sortants d'une Université de Cracovie rénovée, manquait de ressort et ne s'affirmait que dans les grandes villes et dans les villes d'importance moyenne. S'il y eût sermons et que le thème biblique y fût abordé, c'était par référence à la lecture de l'Épitre et de l'Évangile propres au dimanche donné; c'est dire que leur propos était autre que de stimuler un intérêt soutenu pour l'Écriture Sainte.

La lecture individuelle ou collective de la Bible n'était pas au programme des confréries religieuses qui regroupaient les citadins contribuant le plus activement à la piété collective ${ }^{3}$. En effet, la lecture de la Bible était étrangère au modèle de la dévotion des laïcs, préconisé et encouragé par l’Église.

Et qu'en fut-il des intellectuels? La première génération des savants de l'Université de Cracovie, formée dans sa majorité à Prague, et la deuxième, formée déjà sur place, faisaient aux études bibliques une part belle dans leur réflexion théologique. Ceci tenait pour une part au programme de l'enseignement universitaire où l'étude de la Bible constituait un thème suivi et important, ce qui faisait qu'il s'écrivait à l'Université de nombreux commentaires bibliques ${ }^{4}$. Outre cette production "de masse“, peu originale pour la plupart, il y a lieu de distinguer un courant qui accentuait le rôle de l'Écriture Sainte dans la formation intérieure de l'homme, et qui, en même temps, critiquait l'exégèse traditionnelle, formelle, des textes bibliques. Selon Nicolas de Kozłów (mort en 1444), „L'Écriture Sainte, il faut l'enseigner de manière à émouvoir l'homme par des exemples plutôt que par l'argumentation, par des promesses plutôt que par des raisons, par la piété plutôt que par des définitions" 5 . Les théologiens de Cracovie mettaient en vedette les valeurs de morale pratique à tirer de l'étude de la Bible et de son exposé pertinent. Par moments, leurs idées se rapprochaient d'une façon très marquée du modèle de devotio moderna ${ }^{6}$. Le perfectionnement de l'homme devait passer selon eux, par un retour aux sources du christianisme: l'Écriture Sainte et les sacrements. S'ils

${ }^{3}$ Hanna Zaremska, Les confréries religieuses et les corporations à Cracovie aux XIVe-XVe siècles, dans: Questiones Medii Aevi, t.2 (Varsovie 1981) 151-171.

${ }^{4}$ Stanistaw Wielgus, Die mittelalterlichen polnischen Bibelkommentare, in: Probleme der Bearbeitung mittelalterlicher Handschriften (Wolfenbütteler Forschungen 30, 1986) 277-299. 5 Marian Rechowicz, Po założeniu Wydziału Teologicznego w Krakowie, dans: Dzieje teologii katolickiej w Polsce, t.1 (Lublin 1974) 120; Stanisław Wielgus, Średniowieczna łacińskojęzyczna biblistyka polska (Lublin 1992) $54 \mathrm{f}$.

6 Pawet Sczaniecki, Nauka o modlitwie, dans: Dzieje teologii, t.1, 331-332; Wielgus, Średniowieczna biblistyka, 75, 95 . 
percevaient le rôle important à jouer par la Bible, c'est toujours par l'intermédiaire d'un prêtre, enseignant ou prédicateur, qu'il devait s'exercer. En tout cas, leur fait n'était nullement de recommander aux laïcs lettrés une étude libre de la Bible ni d'insister sur la nécessité d'en promouvoir une traduction en polonais, propre à servir à des foules de fidèles. Telle fut aussi la position des théologiens plus nettement ouverts que les autres, sur les plus fervents dans la piété parmi les laïcs. Mathieu de Cracovie fut l'un des rares en Pologne à considérer les avantages d'une Communion fréquente pour les pieux laïcs ${ }^{7}$, mais lui aussi passait sous silence la question d'une mise à leur disposition des textes de la Bible.

L'opposition à un péril hussite exagéré, influença d'une manière décisive, et pour bien longtemps, la position de l'Église en Pologne sur l'accès des laïcs à la Bible. Il s'agissait aussi bien d'une traduction de l'Écriture Sainte en polonais que de la circulation des textes du Nouveau Testament in vulgari. Le processus était complexe et à plus d'un niveau.

Dans le premier quart du XVe siècle, les autorités ecclésiastiques en Pologne décrétèrent une recherche de livres d'auteurs condamnés pour hérésie, et de tous textes suscitant le soupçon quant à leur orthodoxie. Les „Remedia contra hereticos“ inclus dans les statuts synodaux de l'archevêque de Gniezno, Mikołaj Trąba $(1420)^{8}$ ordonnaient aux évêques résidentiels des diocèses polonais de contrôler les livres en possession du clergé paroissial pour vérifier „s'ils ne comprenaient pas quelque chose de suspect “ et de procéder éventuellement à leur confiscation. Une ordonnance postérieure de son sucesseur, l'archevêque Wojciech Jastrzębiec (1430) insistait sur le contrôle des lectures du clergé et des sermonnaires qui leur servaient comme modèles pour leurs propres sermons ${ }^{9}$. Aux époques postérieures aussi était de mise la poursuite des oeuvres d'hérésiarques: Hus, Wiclef, Iakoubek de Stř́ibro, et des livres énigmatiquement qualifiés d' „hérétiques“ ou de „suspects" 10 .

La première moitié du XVe siècle vit l'afflux en Pologne d'un certain nombre de textes en tchèque. Nous en savons fort peu, la plupart de ces textes ne s'étant pas conservés. En faisaient partie des textes de provenance hussite, et ce sont eux qui mirent en suspicion tout ouvrage en tchèque. Parmi ces derniers, il put se trouver en Coujavie une Biblia bohemica lingua ${ }^{11}$. Bien entendu, les autorités ecclésiastiques en Pologne avaient vent de l'importation de textes hussites ou radicalement

7 Mateusz z Krakowa, Opuscula theologica dotyczące spowiedzi i komunii, ed. par Wtadystaw Seńko et Adam Ludwik Szafrański (Textus et studia historiam theologiae in Polonia exultae spectantia, vol. II fasc. 1) (Warszawa 1974) 354-409.

8 Statuty synodalne wieluńsko-kaliskie Mikołaja Trąby z r. 1420 éd. par Adam Vetulani, Jan Fijatek (Kraków 1915-1951) $94 \mathrm{ff}$.

9 Jakub Sawicki, Concilia Poloniae, t.10 (Wrocław 1973) 127.

${ }_{10}$ Edward Potkowski, Krytyka i reforma. Teksty publicystyki kościelnej w Polsce XV w., dans: Literatura i kultura późnego średniowiecza w Polsce, sous la dir. de Teresa Michatowska (Warszawa 1993) 191 f.; Ordinationes sub regimine Petri de Bnin, Episcopi. Anno 1487 editae, dans: Statuta synodalia dioecesis Wladislaviensis et Pomeraniae, collegit Z. Chodyński (Warszawa 1890) 27.

11 Stanisław Wielgus, Obca literatura biblijna w średniowiecznej Polsce (Lublin 1990) 45. 
réformateurs, comme elles savaient aussi que de tels textes étaient copiés et recopiés et sûrement aussi linguistiquement remaniés et mis en forme pour les rendre accessibles au lecteur polonais. Avec la présence d'un livre in vulgari, qui le rendait utilisable pour les laïcs lettrés, le risque perçu de propagation de l'hérésie prit une dimension nouvelle. Il y eut aussi au sein de l'Église, une prise de conscience du risque d'erreurs nouvelles dans la foi à la suite d'une lecture non contrôlée de textes religieux par des laics non avisés.

Avant même le milieu du XVe siècle, s'était affirmée en Pologne une demande d'écrits religieux en polonais, et en premier lieu de traductions de textes bibliques. Nous ne sommes pas en état de mesurer la taille de ce phénomène. En fait foi cependant une prise de position attribuée à un canoniste de Cracovie, Jakub fils de Parkosz de Żurawica, auteur d'un traité sur l'ortographe polonaise (vers 1440). L'introduction à ce traité comprend, outre des considérations sur l'importance d'une ortographe correcte, une polémique avec le point de vue attribuant a priori des erreurs contre la foi à tout écrit religieux in vulgari, et mettant en garde contre le danger que présente leur lecture par les laïcs. C'est donc pour cause que surgit la question de l'Écriture Sainte entre les mains des laïcs. Le docte auteur affirmait que „ce n'est ni la chose en soi ni l'instrument qui est louable ou blâmable, mais bien l'usage qui en est fait: pertinent ou abusif. S'il t'arriva de choir à force d'avoir abusé du vin, la faute n'en fut pas au vin, mais à toi-même. S'il en fût autrement, il eût fallu renoncer à l'Écriture Sainte, étant donné que les hérétiques eux aussi s'en prévalent à l'appui de leurs fausses thèses (...) Mais s'ils sont à blâmer, ce n'est pas pour adhérer à l'Écriture Sainte, mais bien pour en avoir une intelligence erronée"12. La prudence de cette prise de position saute aux yeux. Son auteur n'avait d'ailleurs rien à voir avec ceux qui se servaient des Évangiles à des fins d'une propagande hussite. Il est à croire qu'il avait à l'esprit les milieux tant soit peu instruits de la noblesse et des citadins, lecteurs en puissance des écrits religieux en polonais. Tout au long du XVe siècle, ils ne disposaient pas d'une traduction complète de la Bible. Certes, il y eut des traductions de l'Évangile et des Actes Apostoliques qui circulaient, mais l'on ignore dans quelle mesure elles étaient complètes et habilitées. C'est d'une aire de culture et d'idées et d'un milieu entièrement différents que relevait la première traduction polonaise de la Bible - nous ignorons si elle était complète - faite au milieu du XVe siècle sur recommandation de la reine Sophie, dernière épouse du roi Ladislas Jagellon ${ }^{13}$.

12 Jakuba Parkosza, Traktat o ortografii polskiej, éd. par M. Kucata (Warszawa 1985) 61: „Absit autem hec paralogismi estimacio, ne modico fermenti presentis masse corrumpatur intencio. Non enim res vel instrumentum in se proprie est laudis vel vituperii receptivum, sed tantum eius usus vel abusus. Vini enim non labes, sed tua, si post vina labes. Ex simili itaque Sacra pagina deberet pretermitti racione, qua ipsi heretici suas muniunt intenciones, ut patet in tractatu venerabilis Benedicti, decretorum doctoris, abbatis Marsilie, ubi quemlibet articulum per Sacram Scripturam demonstrant, per hoc autem, quod demonstrant, non redarguuntur heretici, sed quod demonstratam indigeste capiunt, vituperium non ammitunt."

13 Maria Kossowska, Biblia w języku polskim, t.1 (Poznań 1968) $68 \mathrm{f}$.; Michatowska, Średniowiecze, 569-574. 
Il n'est guère facile de répondre à l'interrogation sur l'importance des échos de l'hussitisme pour la généralisation de l'Écriture Sainte parmi les laïcs. C'est qu'il faut avoir à l'esprit que rien que le fait de l'utilisation individuelle d'un texte biblique in vulgari ou de le mettre en circulation, faisait peser sur les individus et les milieux impliqués un soupçon d'hérésie. C'est ce qui occulte le tableau réel des influences hussites en Pologne et en même temps rend difficile une évaluation valable de leur impact sur la généralisation de la Bible en Pologne.

Entre la quatrième la sixième décennie du XVe siècle, l'influence hussite en Pologne rayonnait à partir de petits foyers d'adhérents et de sympathisants de l'hussitisme dans l'ouest et dans le nord du Royaume de Pologne (Grande Pologne, Coujavie, Masovie $)^{14}$. Un tel foyer s'articulait autour d'un notable local en conflit avec l'Église, pratiquant l'utraquisme avec les membres de sa famille, les gens de sa petite cour et ses domestiques, et accordant sa protection aux prêtres hussites.

L'exposé de l'Écriture Sainte dans l'esprit hussite fait l'objet de l'accusation consignée dans un procès-verbal des interrogatoires d'hérétiques dans la ville privée de Zbąszyń en Grande Pologne (1439). Un nommé Miklas de Gniezno recevant la communion sub utraque speciae se vit inculpé par la cour épiscopale, d'avoir procédé devant des paysans dans une taverne de village, à une explication tendancieuse de l'Écriture Sainte, en défigurant le vrai sens. Et ce faisant, il consultait publiquement le livre sensu Bohemico scriptum ${ }^{15}$, soit indubitablement de provenance hussite. Il se peut qu'il s'agît de commentaires au Nouveau Testament ou d'un tout autre ouvrage qui nous reste pourtant inconnu pour avoir été ultérieurement détruit sur ordre des autorités ecclésiastiques.

Ce qui pourtant peut nous intéresser davantage, ce sont les dépositions des partisans réels ou présumés de l'hussitisme de la bourgade de Pakość en Grande Pologne (1455) ${ }^{16}$. Dans un procès où comparaissait comme accusé un nommé Stanislas, magister scholae du lieu, se succédèrent comme témoins des laïcs et des prêtres, des membres du service d'église, des habitants des villes et des nobles. Des femmes aussi eurent à déposer. Tous, ils avaient contribué de diverse façon à faire circuler des livres écrits en polonais ou comprenant des textes mixtes: polonais et latins. Quelques-uns des textes avaient été copiés dans un petit scriptorium près l'école du lieu. Les témoins qui plutôt niaient avoir lu ces livres (en déclarant ne les avoir que provisoirement gardés, transportés, bref, en soulignant le caractère passager de leur accès aux livres incriminés), disaient n'en avoir retenu que les incipit en polonais ou des phrases détachées de leur contexte. Ces dernières auraient peut-être révélé quelquefois soit un contenu hussite soit rien que des observations

${ }^{14}$ Jaromir Mikulka, Polské země a herese v době před reformací (Praha 1969) 63-84; Stanistaw Bylina, Les influences hussites en Pologne et sur les territoires ethniquement russiens du Grand-Duché de Lituanie, dans: Ricerche Slavistiche 41 (1994) 167-176.

15 Voir un texte publié par Józef Nowacki, Biskup poznański Andrzej Bniński w walce z husytami Zbąszynia, dans Roczniki Historyczne 10 (1934) 268 ( $\mathrm{N}^{\circ} 5$ 1439).

16 Acta capitulorum nec non iudiciorum ecclesiasticorum selecta, edidit Boleslaw Ulanowski, t.2 (Kraków 1902) Nr. 524, 198-199. Voir aussi: Edward Potkowski, Le livre manuscrit - la société - la culture dans la Pologne du bas Moyen Age (XIVe-XVe s.)(Warszawa 1987) 110. 
critiques d'esprit réformateur. D'autres témoins ne gardaient en mémoire que le fait que "quendam librum vulgariter ad toto scriptum" ou encore le fait que le volume était relié de rouge. Les interrogations sur la possession et la diffusion des oeuvres hérétiques s'assortissaient d'incriminations et de soupçons imputant aux personnes impliqués dans ce procès, un séjour en Bohême, des intelligences avec des hussites tchèques, la pratique de l'utraquisme.

C'est dans un contexte d'accusations, de suspicions et de dénonciations que la cour examinait l'affaire de la circulation des textes bibliques. Chez l'accusé principal on aurait vu des „epistolas dominicales et Evangelia in vulgari scripta“ (selon la formule d'un des témoins, c'était "novum testamentum vulgariter scriptum“) ${ }^{17}$. L'on apprend aussi que tel exemplaire ou un autre du Nouveau Testament faisait l'objet d'intérêt de représentants de la population urbaine et de femmes nobles. Et l'on tombe à l'occasion sur le terme libri feminarum par lequel on entendait les livres religieux en polonais avec une traduction du Nouveau Testament ${ }^{18}$. Ce qui frappe, c'est la perception de cette littérature parmi les femmes nobles de la vaste zone limitrophe entre la Grande Pologne et la Coujavie. C'est dire que l'intérêt porté à l'Écriture Sainte dépassait le cadre de communauté locales de sympathisants de l'hérésie hussite. Jusqu'à un certain grade social, il s'affirmait par-dessus les clivages sociaux chez les laïcs lettrés, citadins de petites villes ou habitants de gentilhommière au-dessous en tout cas d'une noblesse titulaire de charges locales.

Rares sont les cas documentés d'une haute culture religieuse par l'approche de l'Écriture Sainte hors de l'Église, d'où, par conséquent, la rareté de la faculté d'évaluation critique des réalités de la vie de l'Église. Les progrès déjà sensibles vers le milieu du XVe siècle, de l'initiation des laïcs à l'Écriture Sainte, allaient de pair avec un anticléricalisme - pour des raisons diverses - de nobles et des citadins ${ }^{19}$. Il se manifestait entre autres par une insatisfaction déclarée du contenu des sermons dominicaux et par un persiflage de prêtres insuffisamment instruits. Il y eut un sens du besoin de la conformité de la conduite des prêtres aux principes évangéliques (ce qui n'excluait pas une tolérance pour le mode de vie laïc de certains d'entre eux).

Au niveau de la paroisse, naissait un ferment, certes encore éloigné d'une contestation religieuse consciente, mais non sans importance pour l'acceptation ultérieure de courants réformateurs. Un quart de siècle avant la Réforme, il y a des gens qui savent déjà faire valoir des arguments tirés de l'Évangile dans leurs âpres disputes avec le clergé, alors qu'auparavant des documents ne nous mettent que sur la piste de ceux qui, forts de leur lecture du Nouveau Testament, se montrent portés à démontrer publiquement l'ignorance du bas clergé paroissial. En 1450, un nommé Jan Żołądek, modeste tailleur de la ville masovienne de Łomża, intervenait publiquement les trois jours consécutifs de Pâques, en critiquant les sermons des

17 Acta capitulorum, t.2, 199.

18 Ebd., t.2, 198.

19 Il faut consulter les observations de Jerzy Wiśniowski, dans: Storia del cristianesimo in Pologna, sous la dir. de Jerzy Ktoczowski (Bologna 1980). 
vicaires locaux ${ }^{20}$. Le grief qu'il retenait contre ces prêtres était qu'en expliquant aux fidèles les descriptions évangéliques de la résurrection du Christ, ils avaient fait sauter des détails importants (le fait, par exemple, que le Sauveur apparut à Marie-Madeleine „tanquam ortulanus cum fossorio alias rydlem“), ils n'avaient pas fait la distinction entre Apôtres et disciples, et n'avaient pas su reprendre avec précision les paroles du Christ, rapportés par l'Évangile. Mettons de côté le niveau des connaissances du fervent et petulant artisan. Les objections qu'il avança traduisaient sa certitude du prestige exceptionnel et du poids d'un texte de l'Évangile découvert par un laïc sans intermédiaire d'ecclésiastiques. Les vicaires ont porté plainte en demandant au tailleur des dommages-intérêts pour offense et pour propos diffamants; quant à la cour ecclésiastique, elle y entrevit un cas plus grave d'attitude chez la partie adverse: tanquam pestifera et fidei katholice suspecta ${ }^{21}$. Aux yeux des ecclésiastiques, ce procès confirmait le risque qu'il y aurait à laisser accéder les laïcs à la lecture de l'Écriture Sainte. Sans préciser la nature de l'erreur de l'accusé, la cour replaça son attitude dans un contexte très voisin de l'hérésie.

Les gens qui, dans leurs polémiques avec le clergé, fondaient leurs arguments sur l'Écriture Sainte, se voyaient aisément accusés d'hérésie, sans que cela fût lourd de conséquences pour les personnes de haut rang social. Au seuil de la Réforme, c'est non sans ostentation que l'on affichait des opinions contraires à la position de l'Église. „Nombreux sont les hérétiques, et moi j'en suis“ - une telle déclaration fut faite dans un procès instruit par une cour ecclésiastique. Les attitudes de ce genre formaient un courant non sans importance pour les faits ultérieurs, mais faible et marginal dans le christianisme polonais de la seconde moitié du XVe siècle.

La généralisation de la cuture écrite dans la période considérée sollicita de larges couches de nobles et de habitants des villes 22 . Elle eut pour conséquence la lecture par les lettrés de textes religieux en polonais, parmi lesquels l'Écriture Sainte tenait une place qui est toutefois difficile à mesurer. Cette initiation des laïcs aux Écritures procédait par des chemins indirects: sermons, chants religieux créés et propagés par les franciscains observantins ${ }^{23}$, peintures murales etc. Par l'intermédiaire d'une littérature pour le grand public et de la peinture, l'apocryphe se gagnait des lecteurs en nombre croissant. Désireuse de détails dont l'Évangile omet de se faire l'écho, de la vie du Christ, de la Sainte-Vierge et des saints, l'imagination collective en trouvait dans les apocryphes ${ }^{24}$. La Réforme européenne du XVIe siècle trouva une société polonaise religieusement sous-instruite et non préparée à des confron-

\footnotetext{
20 Acta capitulorum, t.3 (Kraków 1905) 7-8 ( $\left.\mathrm{N}^{\circ} 16,17,18\right)$.

21 Acta capitulorum, t.3 $8\left(\mathrm{~N}^{\circ} 18\right)$.

22 Voir le remarques de Potkowski, Le livre manuscrit, 117-122.

23 Jerzy Kloczowski, The Brothers Minor in Medieval Poland, dans: Du même, La Pologne dans l'Eglise médiévale (Aldershot 1993) 107; Jacek Wiesiotowski, Problemy społeczne klienteli bernardynów poznańskich na przełomie XV/XVI w., dans: Franciszkanie w Polsce średniowiecznej, t.1, sous la dir, de Jerzy Ktoczowski (Lublin 1983) 337-366.

24 Michatowska, Średniowiecze, $616 \mathrm{ff}$.
} 
tations idéologiques incontournables. Il est à présumer que ceci tenait pour une part à une connaissance insuffisante et superficielle du Nouveau Testament.

La Réforme apporta en Pologne une situation nouvelle qui faisait cependant une place à la continuation de phénomènes ayant existé antérieurement. Ce qui fut nouveau c'était, selon un bibliste polonais, un "véritable épanouissement des traductions qui ne se produisit qu'au XVIe siècle, à la faveur, d'une part, de l'essor de l'imprimerie, et d'autre part, de l'impact des idées humanistes“25. Il s'y ajoute encore un troisième facteur: la rivalisation confessionnelle des catholiques avec les protestants et le besoin qui en découlait pour chacune des deux parties de disposer de ses propres traductions (les seules crédibles). Il s'agissait en premier lieu des livres du Nouveau Testament dont une traduction complète devait cependant attendre longuement de voir le jour, alors que c'est le psautier qui, invariablement, jouissait d'une grande popularité auprès des ecclésiastiques et des laïcs. Ce recueil d'une belle poésie religieuse d'un accès plutôt difficile, était seul à n'avoir jamais préoccupé les gardiens de l'orthodoxie.

Or, l'Église catholique en Pologne dont le prestige ne cessait d'être grand, malgré les pertes causées par la Réforme, entrevoyait toujours du péril dans une lecture incontrôlée du Nouveau Testament en traduction polonaise. Cette fois, ce "péril“" aurait favorisé la propagation du luthéranisme. Ainsi que d'ailleurs l'a fait observer Janusz Tazbir, plutôt qu'en persécution des gens, la lutte de l'Église en Pologne contre la Réforme consistait en poursuite des écrits hérétiques (Bibles protestantes, recueils de cantiques etc. ${ }^{26}$. „Le feu des livres brûlés était le seul qu'attisaient les luttes religieuses en Pologne. " 27 D'une manière analogue à ce qui se voyait à l'époque hussite, c'est à un degré minime que des représailles pouvaient s'exercer contre une noblesse dissidente, à l'abri du privilèges nobiliaire. Dans des conditions nouvelles, la noblesse se mit à réclamer vigoureusement un libre accès à la Bible: "que les prêtres ne nous interdisent pas de faire imprimer en polonais des livres d'histoire, des chroniques, nos lois à nous et en particulier la Bible" - lisonsnous dans la résolution de la diétine des nobles de Środa Wielkopolska (1534) ${ }^{28}$. L'identification est complète de la lutte pour la liberté de l'impression en langue nationale avec celle pour un libre choix de sa religion, considéré comme une extension des droits revenant à la noblesse.

Le milieu du XVIe siècle vit la parution de deux traductions en polonais du Nouveau Testament, d'abord protestante à Königsberg (1553), dûe au mécénat du prince de Prusse Albrecht, puis, catholique à Cracovie (1556). Ce dernier devait offrir un contrepoids à la popularité du texte dissident. L'on savait d'ailleurs que chaque traduction nouvelle offrait matière à des disputes dogmatiques nouvelles.

25 Wtadystaw Smereka, Biblistyka polska (wiek XVI“XVIII), dans: Dzieje teologii, t.2 pars 2 (Lublin 1975) 232.

26 Janusz Tazbir, Rola żywego słowa w propagandzie wyznaniowej, dans: Du même, Szlachta i teologowie (Warszawa 1987) 96.

${ }_{27} \mathrm{Du}$ même, A State without Stakes. Polish religious Toleration in the Sixteenth and Seventeeth Centuries (Warsaw 1973) 46.

${ }^{28} \mathrm{Du}$ même, Reformacja jako ruch umysłowy, dans: Szlachta i teologowie, 35. 
Enfin, au bout de quelques années nouvelles, parut une traduction "catholique“ complète en polonais de l'Écriture Sainte, la Bible dite de Léopolite (Cracovie 1561). Tout en soulignant la grande portée de la Bible dans la vie de chaque chrétien, la préface n'en reprend pas moins le vieil avertissement contre une étude par trop libre de l'oeuvre inspirée.

Il est temps de conclure rapidement. Le problème de l'accès des laïcs à l'Écriture Sainte hors de la liturgie célébrée en l'église surgit en Pologne tardivement: au XVe siècle seulement. Ce retard, on peut l'évaluer sous plus d'un angle. Considéré globalement, il fut la conséquence d'un retard de développement de la culture religieuse. Dans une dimension plus restreinte, l'on peut s'interroger sur les conséquences de l'absence de mouvements hérétiques et radicalement réformateurs au XIIIe-XIVe siècles.

Ainsi que nous l'avons dit précédemment, le fait que, dès le début du XVe siècle, les théologiens universitaires s'étaient tournés d'une façon très perceptible vers l'Écriture Sainte, constituait un phénomène interne d'Église, sans retombées aucunes hors de l'état ecclésiastique. Le problème de l',initiative du peuple en matière de foi (magistralement mis en relief par Amedeo Molnár pour la Bohême des XIVe et XVe siècles) ${ }^{29}$ était étranger aux élites intellectuelles et pastorales de l'Église en Pologne. La situation y était d'ailleurs entièrement différente de celle en Bohême où déjà les débuts de l'hussitisme avaient été marqués par une large et profonde adhésion des fidèles à la Bible ${ }^{30}$. En Pologne, un phénomène d'échelle comparable ne se produisit pas. Et - chose très importante - ne s'y affirmèrent ni les circonstances ni le besoin d'une large confrontation avec l'Église sur le plan des idées.

L'hussitisme en Bohême corrobora très fortement la méfiance du clergé polonais à l'égard d'une activité religieuse incontrôlée des laïcs. Le dépistage, puis la destruction ou la confiscation des ouvrages qualifiés d'hérétiques (en particulier d'auteurs hussites), l'incrimination de tous textes ramenés de Bohême, la méfiance envers toute littérature religieuse in vulgari (pas seulement en tchèque, mais également en polonais) ont repoussé les traductions polonaises de l'Écriture Sainte jusqu'au bout d'un chemin jalonné de restrictions. Les appréhensions et la suspicion allaient reparaître au XVIe siècle, dans les circonstances d'une lutte de l'Église contre les dissidents.

Je ne pense pas que le faible courant de contestation religieuse d'esprit hussite qui ne s'affirma que dans quelques-unes des régions de la Pologne, y contribuât grandement à la généralisation de la lecture de l'Écriture Sainte. Il put par contre, favoriser la propagation d'attitudes critiques de mise en regard des vérités et des idéaux évangéliques d'une part avec la réalité de la vie de l'Église d'autre part. De telles tendances, d'un degré différencié de radicalisme, se montraient persistantes

${ }^{29}$ Amedeo Molnár, Aktywność ludu w ruchu reformatorskim. Świadectwo kazań husyckich, dans: Kultura elitarna a kultura masowa w Polsce późnego średniowiecza, sous la dir. de Bronistaw Geremek (Wroctaw 1978) 77-118.

30 Jiři Kejřr, Mistři pražské univerzity a kněží táborští (Praha 1981) 27f.; František Šmabel, Dějiny Tábora, t.1 pars 2 (1422-1452) (České Budějovice 1990) 368, 604 et suiv.; Du même, Husitská revoluce, t. 2: Kořeny české reformace (Praha 1993) 35 et suiv. 
jusqu'à l'époque de la Réforme du XVIe siècle. L'attitude de contestation s'exprimait tant dans les traductions non autorisées de l'Écriture Sainte dont le nombre nous échappe, que dans la lecture quelquefois clandestine de ces textes. A plus forte raison, c'était vrai aussi pour un exposé de l'Écriture Sainte indépendant de l'Église, se rapprochant vraisemblablement, au XVe siècle, d'interprétations hussites. Plus tard, au siècle suivant, quand l'un des réformateurs écrivait que „la Parole Divine et l'Évangile s'annoncent en polonais d'une façon pure dans bien des endroits“"31, c'est uniquement la prédication protestante qu'il avait à l'esprit.

31 Tazbir, A State without Stakes, 46. 